\title{
Supporting Information: Uncloaking the Thermodynamics of the Studtite to Metastudtite Shear-Induced Transformation
}

\author{
Philippe F. Weck ${ }^{*}{ }^{\dagger}$ and Eunja $\mathrm{Kim}^{\ddagger}$ \\ ${ }^{\dagger}$ Sandia National Laboratories, Albuquerque, NM 87185, USA. \\ ${ }^{\ddagger}$ Department of Physics and Astronomy, University of Nevada Las Vegas, Las Vegas, NV 89122, USA. \\ KEYWORDS density functional theory, thermodynamics, actinides, oxides, corrosion
}

S1. Main Vibrational Modes of Studtite, $\left(\mathrm{UO}_{2}\right)\left(\mathrm{O}_{2}\right)\left(\mathrm{H}_{2} \mathrm{O}\right)_{2} \cdot 2 \mathrm{H}_{2} \mathrm{O}$, and Metastudtite, $\left(\mathrm{UO}_{2}\right)\left(\mathrm{O}_{2}\right)\left(\mathrm{H}_{2} \mathrm{O}\right)_{2}$

\begin{tabular}{|c|c|}
\hline $\begin{array}{l}\text { Peak Center Frequency } \\
\quad \text { in } \mathrm{THz}\left[\text { in } \mathrm{cm}^{-1}\right]\end{array}$ & Mode Description \\
\hline \multicolumn{2}{|r|}{$\left(\mathrm{UO}_{2}\right)\left(\mathrm{O}_{2}\right)\left(\mathrm{H}_{2} \mathrm{O}\right)_{2} \cdot 2 \mathrm{H}_{2} \mathrm{O}$} \\
\hline $98.9[\sim 3300]$ & asymmetric $\mathrm{H}_{2} \mathrm{O}$ stretching \\
\hline $92.0[\sim 3070]$ & symmetric $\mathrm{H}_{2} \mathrm{O}$ stretching \\
\hline $48.6[\sim 1620]$ & $\mathrm{H}_{2} \mathrm{O}$ scissoring \\
\hline $26.5[\sim 880]$ & $\begin{array}{l}\text { combo: } \mathrm{H}_{2} \mathrm{O} \text { wagging }+\mathrm{O}=\mathrm{O} \text { stretching }+\mathrm{H}_{2} \mathrm{O} \text { rocking; } \mathrm{H}_{2} \mathrm{O} \text { twisting } \\
+\mathrm{O}-\mathrm{O} \text { stretching; } \mathrm{O}=\mathrm{U}=\mathrm{O} \text { asymmetric stretching; } \mathrm{O}=\mathrm{U}=\mathrm{O} \text { symmetric } \\
\text { stretching }\end{array}$ \\
\hline $21.7[\sim 720]$ & combo: $\mathrm{H}_{2} \mathrm{O}$ wagging $+\mathrm{H}_{2} \mathrm{O}$ twisting \\
\hline $18.4[\sim 610]$ & combo: $\mathrm{OH}$ wagging $+\mathrm{H}_{2} \mathrm{O}$ rocking $+\mathrm{H}_{2} \mathrm{O}$ wagging $+\mathrm{H}_{2} \mathrm{O}$ twisting \\
\hline $11.2[\sim 370]$ & $\begin{array}{l}\text { combo: } \mathrm{O}-\mathrm{U}-\mathrm{O} \text { symmetric and asymmetric stretching }+\mathrm{O}=\mathrm{O} \text { twisting } \\
+\mathrm{O}=\mathrm{O} \text { wagging }\end{array}$ \\
\hline $5.5[\sim 180]$ & complex collective "chain" mode \\
\hline \multicolumn{2}{|r|}{$\left(\mathrm{UO}_{2}\right)\left(\mathrm{O}_{2}\right)\left(\mathrm{H}_{2} \mathrm{O}\right)_{2}$} \\
\hline $100.9[\sim 3360]$ & asymmetric $\mathrm{H}_{2} \mathrm{O}$ stretching \\
\hline $95.8[\sim 3200]$ & symmetric $\mathrm{H}_{2} \mathrm{O}$ stretching \\
\hline $48.9[\sim 1630]$ & $\mathrm{H}_{2} \mathrm{O}$ scissoring \\
\hline $27.5[\sim 920]$ & $\begin{array}{l}\text { combo: } \mathrm{O}=\mathrm{U}=\mathrm{O} \text { asymmetric stretching; } \mathrm{H}_{2} \mathrm{O} \text { rocking }+\mathrm{H}_{2} \mathrm{O} \text { twisting } \\
+\mathrm{H}_{2} \mathrm{O} \text { wagging; } \mathrm{O}=\mathrm{O} \text { stretching }\end{array}$ \\
\hline $23.6[\sim 790]$ & combo: $\mathrm{O}=\mathrm{U}=\mathrm{O}$ symmetric stretching $+\mathrm{H}_{2} \mathrm{O}$ rocking \\
\hline $19.9[\sim 660]$ & combo: $\mathrm{H}_{2} \mathrm{O}$ twisting $+\mathrm{H}_{2} \mathrm{O}$ wagging \\
\hline $15.5[\sim 520]$ & $\mathrm{H}_{2} \mathrm{O}$ twisting \\
\hline $11.2[\sim 370]$ & combo: O-U-O symmetric and asymmetric stretching $+\mathrm{O}=\mathrm{O}$ twisting \\
\hline $6.8[\sim 230]$ & complex collective "chain" mode \\
\hline
\end{tabular}

S2. Summary of the Elastic Properties of Studtite, $\left(\mathrm{UO}_{2}\right)\left(\mathrm{O}_{2}\right)\left(\mathrm{H}_{2} \mathrm{O}\right)_{2} \cdot 2 \mathrm{H}_{2} \mathrm{O}$ 


\section{S2.1. Average Properties:}

\begin{tabular}{|c|c|c|c|c|}
\hline Averaging scheme & Bulk modulus & Young's modulus & Shear modulus & Poisson's ratio \\
\hline Voigt & $K_{\mathrm{V}}=34.742 \mathrm{GPa}$ & $E_{\mathrm{V}}=40.299 \mathrm{GPa}$ & $G_{\mathrm{V}}=15.42 \mathrm{GPa}$ & $V_{\mathrm{V}}=0.30668$ \\
\hline Reuss & $K_{\mathrm{R}}=25.7 \mathrm{GPa}$ & $E_{\mathrm{R}}=29.487 \mathrm{GPa}$ & $G_{\mathrm{R}}=11.265 \mathrm{GPa}$ & $V_{\mathrm{R}}=0.30877$ \\
\hline Hill & $K_{\mathrm{H}}=30.221 \mathrm{GPa}$ & $E_{\mathrm{H}}=34.893 \mathrm{GPa}$ & $G_{\mathrm{H}}=13.343 \mathrm{GPa}$ & $V_{\mathrm{H}}=0.30757$ \\
\hline
\end{tabular}

S2.2. Eigenvalues of the Stiffness Matrix:

\begin{tabular}{c|c|c|c|c|c|}
\hline $\boldsymbol{\lambda}_{\mathbf{1}}$ & $\boldsymbol{\lambda}_{\mathbf{2}}$ & $\boldsymbol{\lambda}_{\mathbf{3}}$ & $\boldsymbol{\lambda}_{\mathbf{4}}$ & $\boldsymbol{\lambda}_{\mathbf{5}}$ & $\boldsymbol{\lambda}_{\mathbf{6}}$ \\
\hline $7.0026 \mathrm{GPa}$ & $10.82 \mathrm{GPa}$ & $13.86 \mathrm{GPa}$ & $24.177 \mathrm{GPa}$ & $41.806 \mathrm{GPa}$ & $119.43 \mathrm{GPa}$ \\
\hline
\end{tabular}

S2.3. Variations of the Elastic Moduli:

\begin{tabular}{|c|c|c|c|c|c|c|c|c|}
\hline & \multicolumn{2}{|c|}{ Young's modulus } & \multicolumn{2}{|c|}{ Linear compressibility } & \multicolumn{2}{|c|}{ Shear modulus } & \multicolumn{2}{|c|}{ Poisson's ratio } \\
\hline & $E_{\mathrm{min}}$ & $E_{\max }$ & $\beta_{\min }$ & $\beta_{\max }$ & $G_{\min }$ & $G_{\max }$ & $v_{m i n}$ & $v_{\max }$ \\
\hline Value & $20.525 \mathrm{GPa}$ & $60.749 \mathrm{GPa}$ & $4.5313 \mathrm{TPa}^{-1}$ & $26.068 \mathrm{TPa}^{-1}$ & $7.0192 \mathrm{GPa}$ & $23.388 \mathrm{GPa}$ & -0.080699 & 0.79495 \\
\hline Anisotropy & \multicolumn{2}{|c|}{2.96} & \multicolumn{2}{|c|}{5.7530} & \multicolumn{2}{|c|}{3.332} & \multicolumn{2}{|c|}{$\infty$} \\
\hline
\end{tabular}

\section{S2.4. Spatial Dependence of Young's Modulus:}
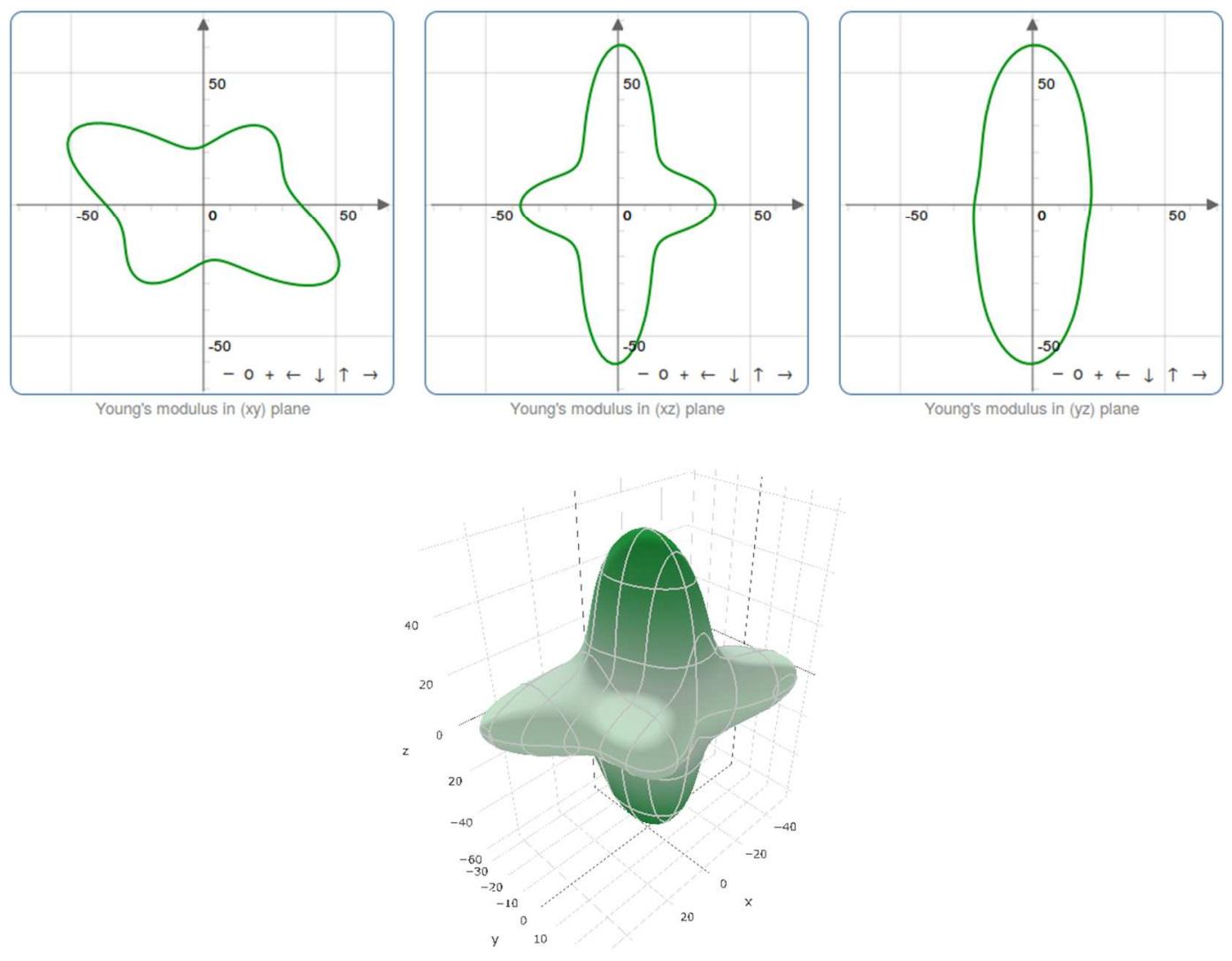
S2.5. Spatial Dependence of Linear Compressibility:

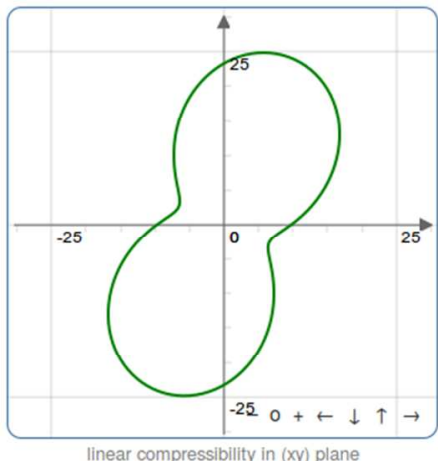

linear compressibility in (ry) plane

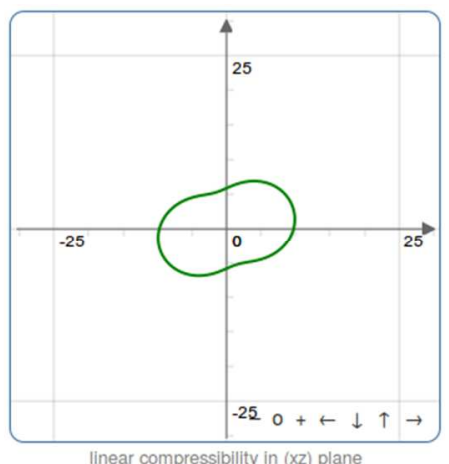

linear compressibility in $(x z)$ plane

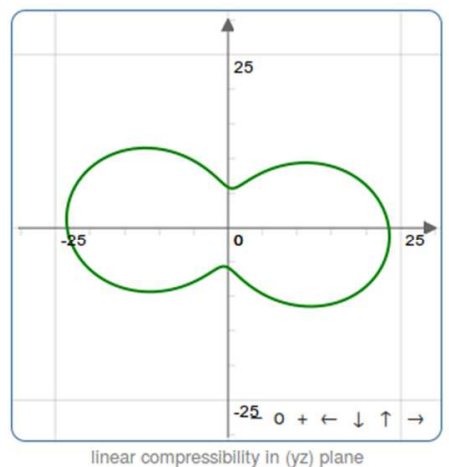

linear compressibility in (byz) plane

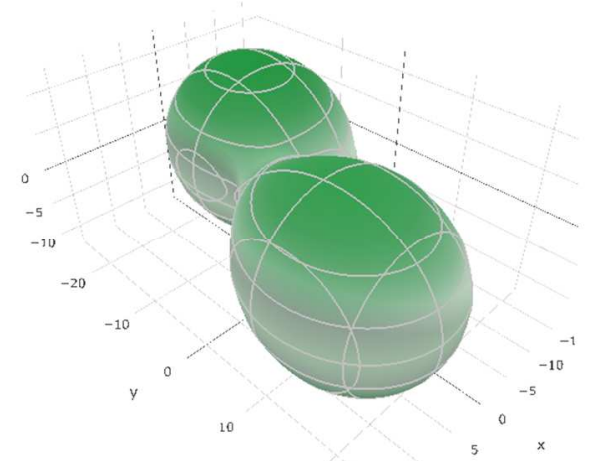

S2.6. Spatial Dependence of Poisson's Ratio:
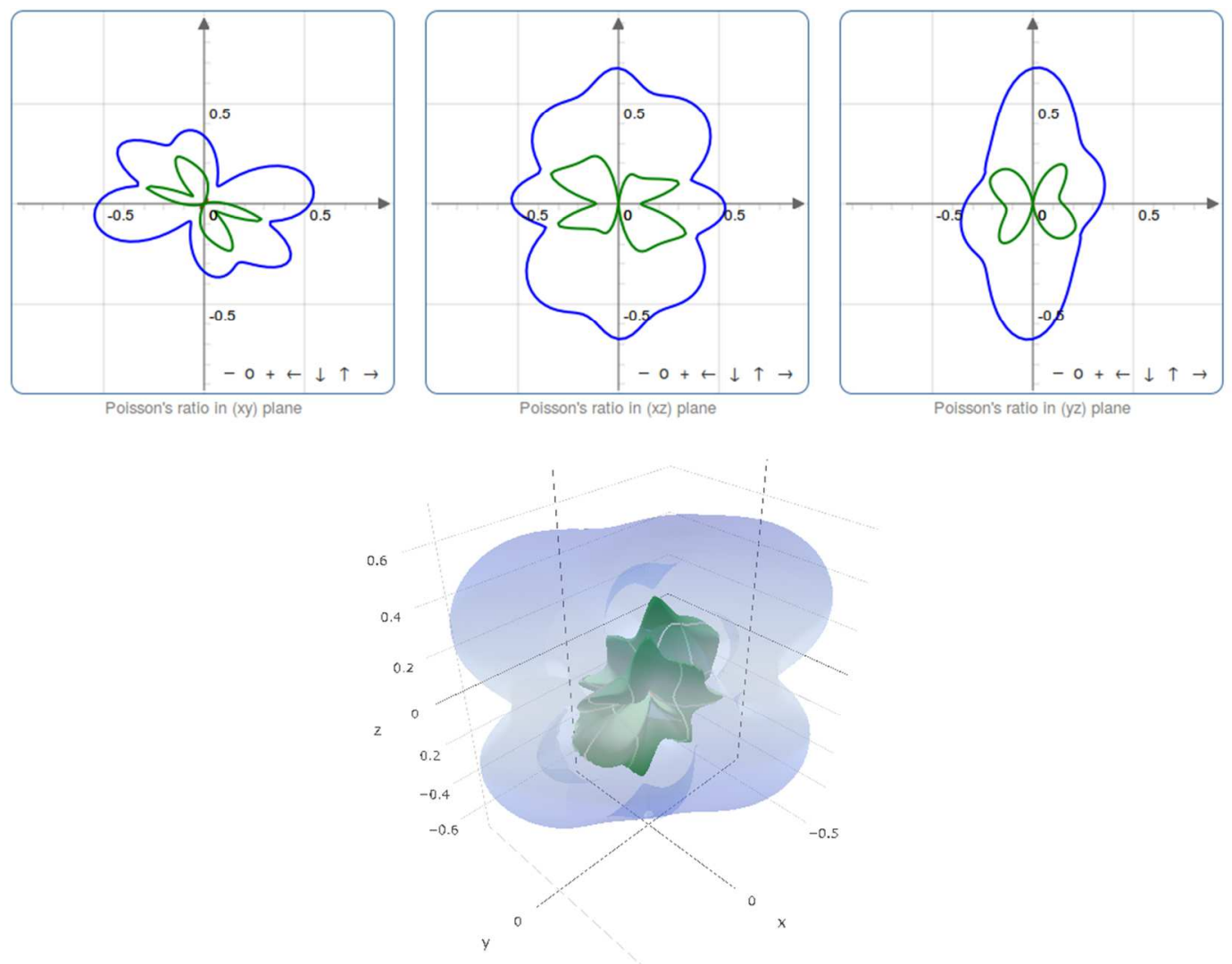

SB 
S3. Summary of the Elastic Properties of Metastudtite, $\left(\mathrm{UO}_{2}\right)\left(\mathrm{O}_{2}\right)\left(\mathrm{H}_{2} \mathrm{O}\right)_{2}$

S3.1. Average Properties:

\begin{tabular}{|c|c|c|c|c|}
\hline Averaging scheme & Bulk modulus & Young's modulus & Shear modulus & Poisson's ratio \\
\hline Voigt & $K_{\mathrm{V}}=44.172 \mathrm{GPa}$ & $E_{\mathrm{V}}=45.052 \mathrm{GPa}$ & $G_{\mathrm{V}}=16.937 \mathrm{GPa}$ & $V_{\mathrm{V}}=0.33002$ \\
\hline Reuss & $K_{\mathrm{R}}=39.271 \mathrm{GPa}$ & $E_{\mathrm{R}}=36.083 \mathrm{GPa}$ & $G_{\mathrm{R}}=13.395 \mathrm{GPa}$ & $V_{\mathrm{R}}=0.34686$ \\
\hline Hill & $K_{\mathrm{H}}=41.722 \mathrm{GPa}$ & $E_{\mathrm{H}}=40.581 \mathrm{GPa}$ & $G_{\mathrm{H}}=15.166 \mathrm{GPa}$ & $V_{\mathrm{H}}=0.33789$ \\
\hline
\end{tabular}

S3.2. Eigenvalues of the Stiffness Matrix:

\begin{tabular}{c|c|c|c|c|c|}
\hline$\lambda_{1}$ & $\lambda_{2}$ & $\lambda_{3}$ & $\lambda_{4}$ & $\lambda_{5}$ & $\lambda_{6}$ \\
\hline $10.183 \mathrm{GPa}$ & $13.137 \mathrm{GPa}$ & $17.799 \mathrm{GPa}$ & $18.39 \mathrm{GPa}$ & $56.609 \mathrm{GPa}$ & $144.05 \mathrm{GPa}$
\end{tabular}

S3.3. Variations of the Elastic Moduli:

\begin{tabular}{|c|c|c|c|c|c|c|c|c|}
\hline & \multicolumn{2}{|c|}{ Young's modulus } & \multicolumn{2}{|c|}{ Linear compressibility } & \multicolumn{2}{|c|}{ Shear modulus } & \multicolumn{2}{|c|}{ Poisson's ratio } \\
\hline & $E_{\min }$ & $E_{\max }$ & $\beta_{\min }$ & $\beta_{\max }$ & $G_{\min }$ & $G_{\max }$ & $v_{\min }$ & $v_{\max }$ \\
\hline Value & $24.493 \mathrm{GPa}$ & $92.818 \mathrm{GPa}$ & $2.7324 \mathrm{TPa}^{-1}$ & $13.989 \mathrm{TPa}^{-1}$ & $8.9431 \mathrm{GPa}$ & $25.037 \mathrm{GPa}$ & 0.024281 & 0.67393 \\
\hline Anisotropy & \multicolumn{2}{|c|}{3.79} & \multicolumn{2}{|c|}{5.1199} & \multicolumn{2}{|c|}{2.8} & \multicolumn{2}{|c|}{27.7558} \\
\hline
\end{tabular}

S3.4. Spatial Dependence of Young's Modulus:
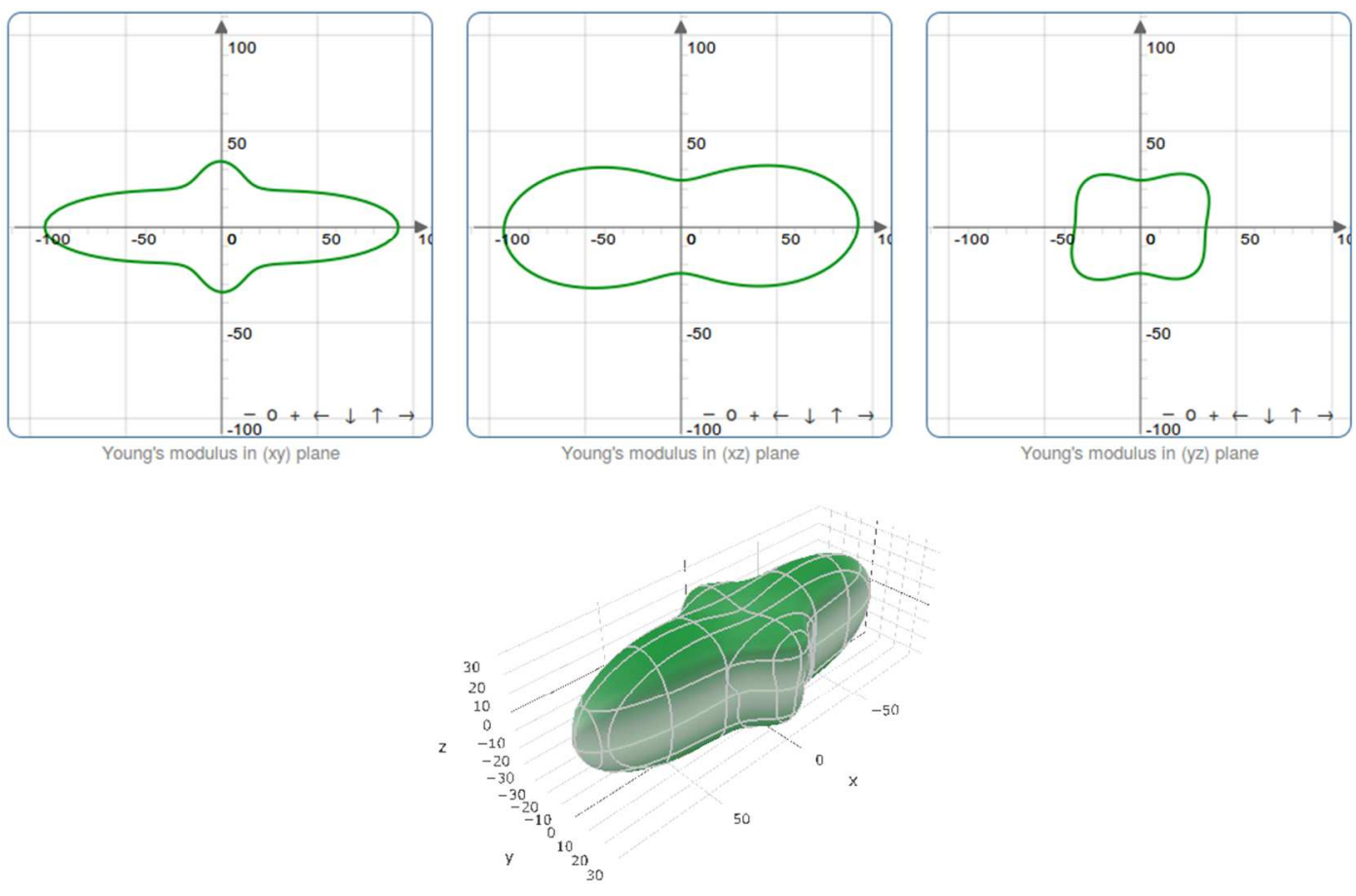
S3.5 Spatial Dependence of Linear Compressibility:
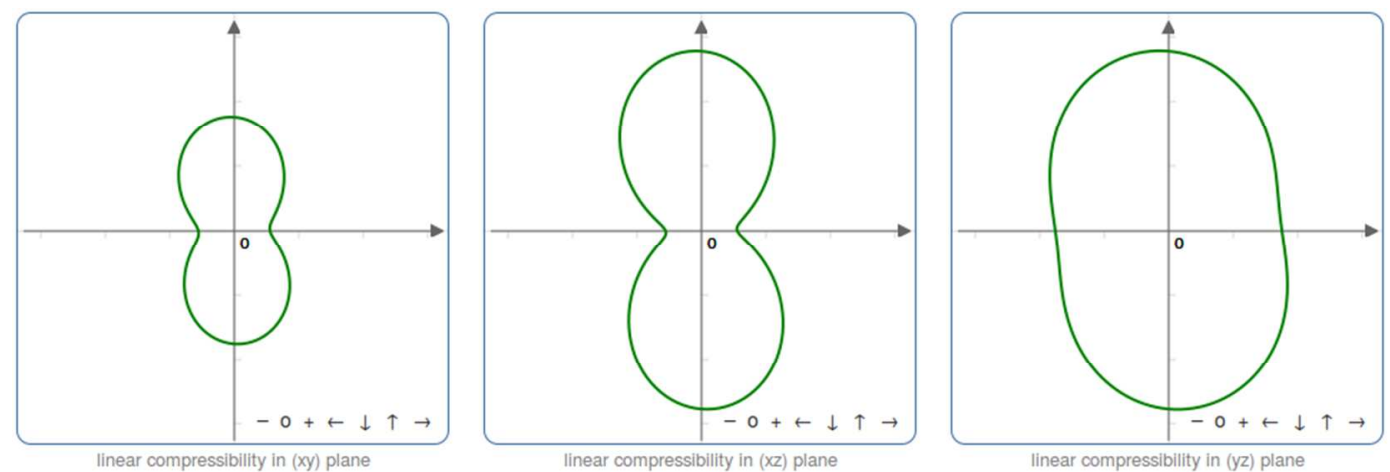

linear compressibility.

linear compressibility in (cz) plane

linear compressibility in (cz) plane

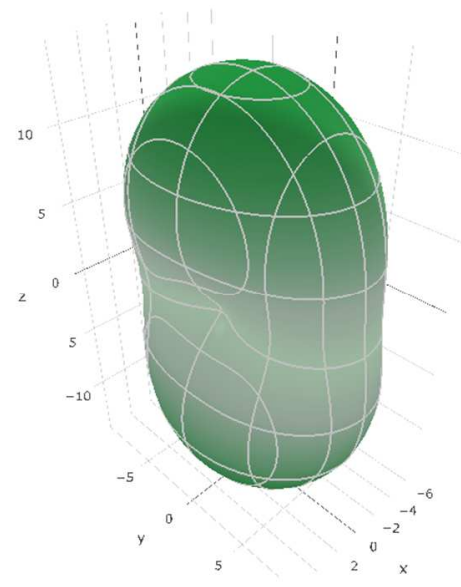

S3.6. Spatial Dependence of Poisson's Ratio:
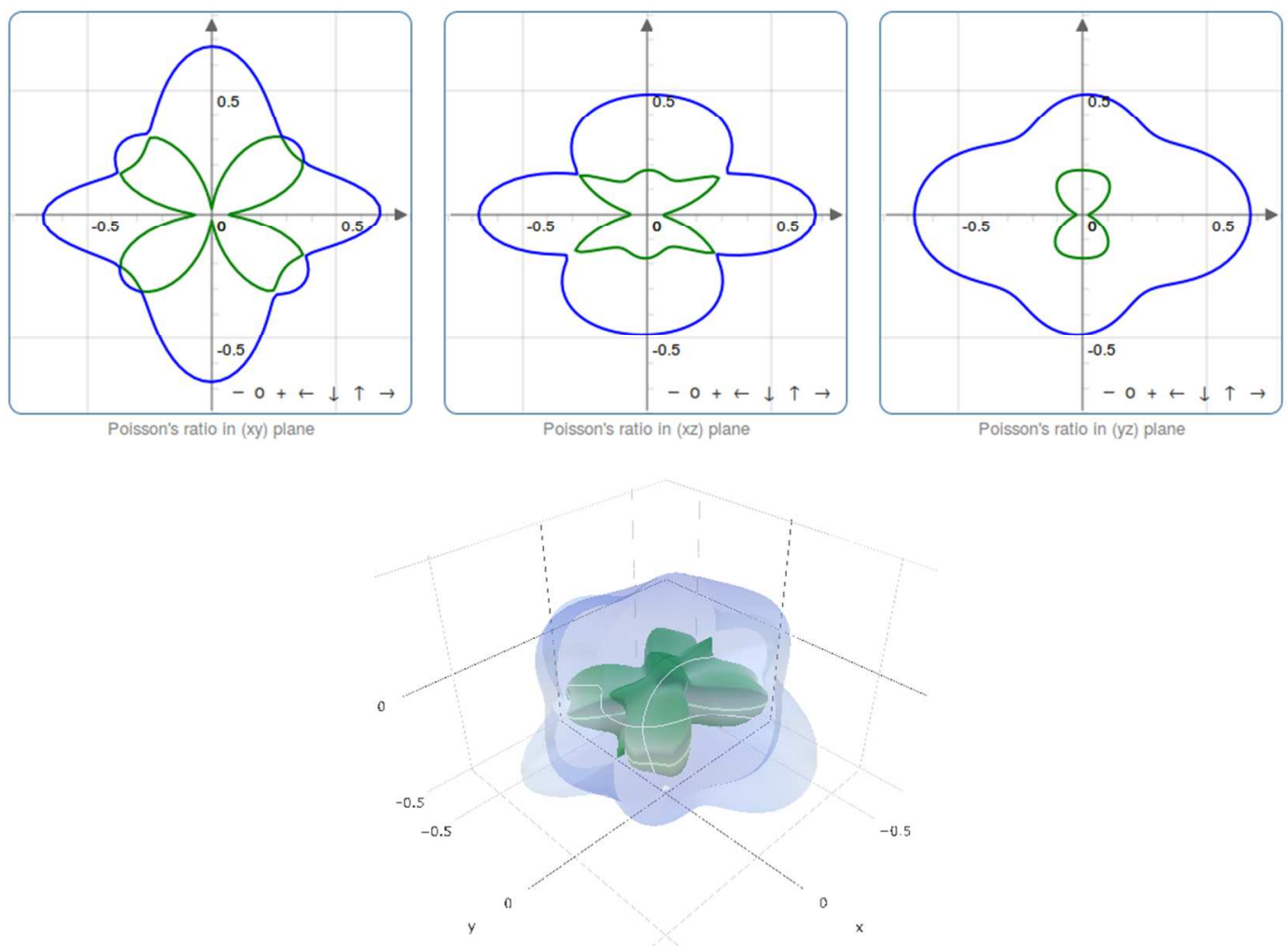

SF 